\title{
Ergonomic thoracic port design for video-assisted thoracoscopic minimally invasive esophagectomy and lymphadenectomy: a preliminary pilot study
}

\author{
Han-Yu Deng ${ }^{1,2 \#}$, Xi Zheng ${ }^{2 \#}$, Guha Alai ${ }^{1}$, Ze-Guo Zhuo ${ }^{1}$, Gang Li ${ }^{1}$, Jun Luo ${ }^{1}$, Yi-Dan Lin ${ }^{1}$ \\ ${ }^{1}$ Department of Thoracic Surgery, ${ }^{2}$ Lung Cancer Center, West China Hospital, Sichuan University, Chengdu 610041, China \\ Contributions: (I) Conception and design: HY Deng, YD Lin; (II) Administrative support: YD Lin, HY Deng; (III) Provision of study materials or \\ patients: G Alai, ZG Zhuo, J Luo; (IV) Collection and assembly of data: J Luo, G Li, G Alai; (V) Data analysis and interpretation: HY Deng, X \\ Zheng, YD Lin; (VI) Manuscript writing: All authors; (VII) Final approval of manuscript: All authors. \\ \#These authors contributed equally to this work. \\ Correspondence to: Yi-Dan Lin. Department of Thoracic Surgery, West China Hospital, Sichuan University, No. 37 Guoxue Alley, Chengdu 610041, \\ China. Email: linyidan.academy@foxmail.com.
}

Background: Video-assisted minimally invasive esophagectomy (MIE) has been widely applied in clinical practice. However, the optimal port design for thoracoscopic esophagectomy and lymphadenectomy has not been well established. Here we introduced our novel ergonomic thoracic port design as well as our novel procedures of lymphadenectomy via tissue interactive retraction and compared its effects with that of conventional port design in this pilot study.

Methods: Patients undergoing McKeown MIE from January 2018 to December 2018 in one surgical team were randomly assigned into the ergonomic port design group and conventional port design group. Data of baseline characteristics, perioperative outcomes, and ergonomic assessment were collected and compared between the two groups.

Results: A total of 70 patients undergoing curative McKeown MIE were randomly assigned and there were 35 patients in each group. The baseline characteristics between the two groups were comparable and well-matched. Moreover, there was no significant difference of number of total dissected lymph nodes, positive lymph nodes and total dissected mediastinal lymph nodes between the two groups. As for perioperative outcomes, there was also no significant difference of in-operating time and blood loss in the thoracic part between the two groups. However, there were significantly less times of forced pause of the surgeon by fatigue during thoracic part in the ergonomic group compared to conventional group (mean time: 1.1 vs. 7.4 , respectively; $\mathrm{P}<0.001$ ) and the symptom score referable to the musculoskeletal system by the surgeon was significantly lower in the ergonomic group than in the conventional group (2.3 vs. 7.6; $\mathrm{P}<0.001$ ). Postoperatively, there was no significant difference of hospital stay duration and rate of complication and 30-day mortality between the two groups.

Conclusions: Our novel thoracoscopic port design and procedures of lymphadenectomy was proved to be feasible and ergonomic, which could be easily mastered by most of the thoracic surgeons.

Keywords: Minimally invasive esophagectomy (MIE); video-assisted; port design; ergonomic

Submitted May 04, 2019. Accepted for publication Jul 24, 2019.

doi: $10.21037 / \mathrm{atm} .2019 .08 .19$

View this article at: http://dx.doi.org/10.21037/atm.2019.08.19 


\section{Introduction}

Esophageal cancer is the eighth most common malignant tumor and the sixth most common cause of death from cancer worldwide (1). Surgery still remains to play an important role in treating esophageal cancer. Currently, minimally invasive esophagectomy (MIE) has been widely utilized as an alternative to open esophagectomy due to the fact that MIE could yield favorable perioperative outcomes with similar oncological outcomes compared with open esophagectomy $(2,3)$. Performing MIE was timeconsuming with an average time duration of about 4 to 6 hours (4). Unlike during open thoracotomy with wide flexibility, the surgeon was required to keep a nearly fixed gesture for a relatively long time due to the limitation of port design during MIE. Usually, thoracoscopic esophagectomy required longer operative time than open esophagectomy (5), which could increase the workload of surgeons causing considerable fatigue (6). Moreover, for thoracoscopic esophagectomy, there was no proposed standard procedure and port design, indicating that improvement still existed for it. Therefore, ergonomic port design was badly needed for MIE. Moreover, difficulties still existed in maintaining an excellent surgical field as well as meticulous procedures $(5,7)$, especially for dissection of lymph nodes around the left recurrent laryngeal nerve (RLN) and subcarinal lymph nodes. Therefore, here we described our ergonomic port design for easy thoracoscopic esophagectomy as well as our novel procedures of lymphadenectomy via tissue interactive retraction for easy lymphadenectomy in this pilot randomized study.

\section{Methods}

\section{Patients}

From January 2018 to December 2018, patients with pathologically confirmed esophageal cancer intended for McKeown video-assisted MIE with two-field lymphadenectomy were randomly assigned into ergonomic incision design group and conventional port design group at one surgical team (Figure 1). Preoperatively, all those patients underwent endoscopy examination, chest computed tomography (CT), abdominal CT, cardiopulmonary function and blood testing routinely, and were evaluated as having a resectable esophageal cancer preoperatively (cT13N0-2M0). Data for analysis included demographic data and comorbidities (hypertension, coronary artery disease, diabetes, and chronic obstructive pulmonary disease) as well as clinical and pathologic TNM stage. Perioperative data of in-operating time, blood loss, postoperative hospital stay, major complications (severe pneumonia, anastomotic leakage, RLN paralysis, esophagotracheal fistula), and 30day mortality rate were also collected and compared. For ergonomic assessment, the pause times of the surgeon forced by fatigue during surgery in the thoracic part were recorded for each patient by one of the authors in the operating room during surgery. Moreover, at the end of the thoracic stage, the surgeon was asked to report his symptoms referable to the musculoskeletal system on a rating score from 1 (uninfluenced) to 10 (maximum fatigue) as described by Shen et al. (6), which was also recorded by the same author.

Our work was approved by institutional review board of West China Hospital, Sichuan University (No. 20170730) and informed consent was obtained from each patient participating in the study.

\section{Surgical techniques}

\section{Position and port design}

For a typical patient, it was placed at the left lateral-prone position, where the patient was first placed left lateral decubitus position and then leaned about 45 degree to the left (Figure 2), which was proved to combine the advantages of both lateral decubitus position and prone position in a previous study providing excellent surgical field (8). The surgeon stood ventrally facing anteriorly to the patient, and the camera surgeon stood at the surgeon's side left to the surgeon, and the assistant stood dorsally to the patient facing posteriorly. Four ports were designed for ergonomic group: one observation port was placed at the seventh intercostal space (ICS) along the scapular line, and one 5 -mm working port was placed at the third ICS about $1.5 \mathrm{~cm}$ posterior to the posterior axillary line and another 10 -mm working port was placed at the fifth ICS along the posterior axillary line, and finally a $5-\mathrm{mm}$ assistant was placed at the ninth ICS about $4 \mathrm{~cm}$ posterior to the scapular line (Figure 2A). In this novel ergonomic port design, the surgeons could complete most of the procedures with his shoulders and arms down, decreasing fatigue of the surgeon during the long-time operation. For conventional port design, which was widely applied in China as previously described $(6,8,9)$, an observation port was placed at the seventh ICS just posterior to the posterior axillary line. Three other ports for the operating instruments were placed as follows: an upper port at the posterior axillary line 


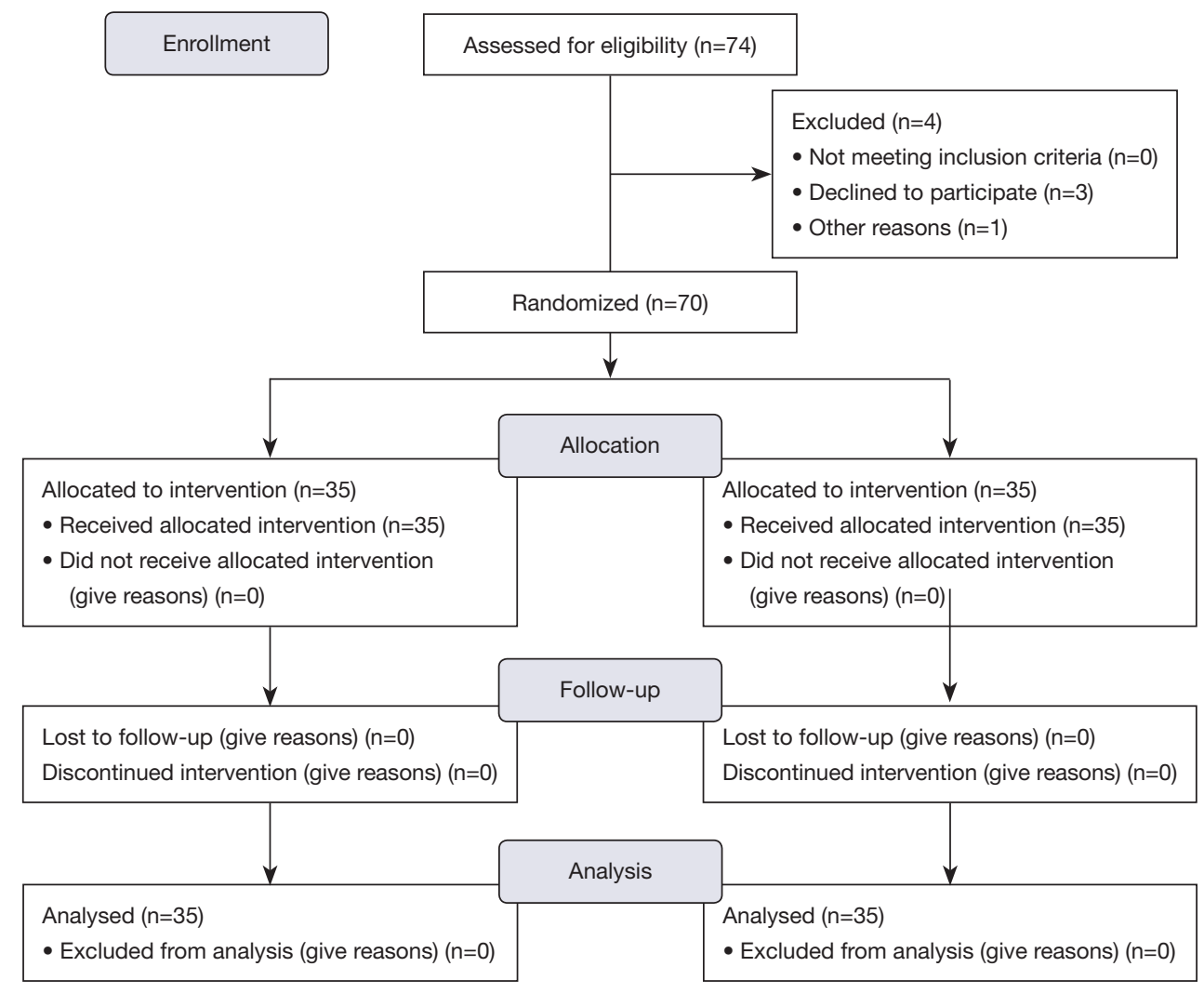

Figure 1 A flow diagram of our pilot study.

in the fourth ICS, a middle port just inferior to the scapula tip, and a lower port at the scapula line in the ninth ICS (Figure 2B).

\section{Mobilization of the esophagus and lymphadenectomy for both ergonomic port design and conventional pot design}

After thoracoscopic examination, the arch of the azygos vein was first identified and ligated by vascular clips at the conjugation of azygos vein with right superior intercostal vein, clipping the azygos vein and right superior intercostal vein as well as the arch of azygos vein respectively to set free for the thoracic esophagus. Then, the thoracic esophagus was mobilized dorsally along the groove between the vertebral body and esophagus below the arch of the azygos vein cranially to the dome of the right thorax while the vagus nerve was identified and the right RLN was carefully protected (Figure 3A). Meanwhile, the right upper mediastinal lymph nodes were also carefully dissected.

After the posterior aspect of the esophagus was dissected, it was pulled laterally and anteriorly by an assistant through the port at the fifth ICS via pulling the surrounding tissues around the esophagus, exposing the left side of the esophagus as much as possible. Because the trachea was attached with the esophagus, both the esophagus and trachea were pulled together via their tissue interactive retraction. Due to the fact that the left mediastinum was deeper in the thorax, the surgeon temporarily utilized the port at the third ICS and port at the ninth ICS to ease the dissection of lymph nodes around the left RLN, which was carefully identified and protected, as well as the left upper mediastinal lymph nodes (Figure 3B).

After bilateral upper mediastinal lymph nodes were dissected, the subcarinal lymph nodes were en bloc dissected along with the esophagus via the interactive retraction of the esophagus as follow: first, the esophagus was pulled laterally and the left main bronchus was exposed, and then the left part of subcarinal lymph nodes was dissected from distal to proximal just below the left main bronchus. Second, the esophagus was pulled up and the gap between the esophagus and the bronchus was exposed, and the right part of subcarinal lymph nodes were also dissected from 

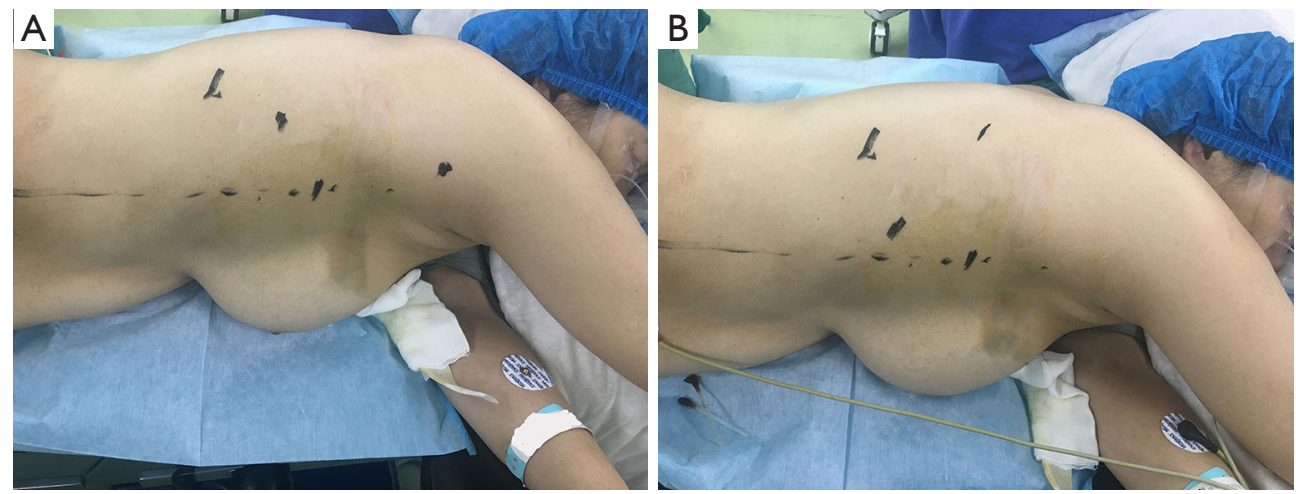

Figure 2 Port design for video-assisted thoracoscopic MIE. (A) Ergonomic port design; (B) conventional port design. MIE, minimally invasive esophagectomy.
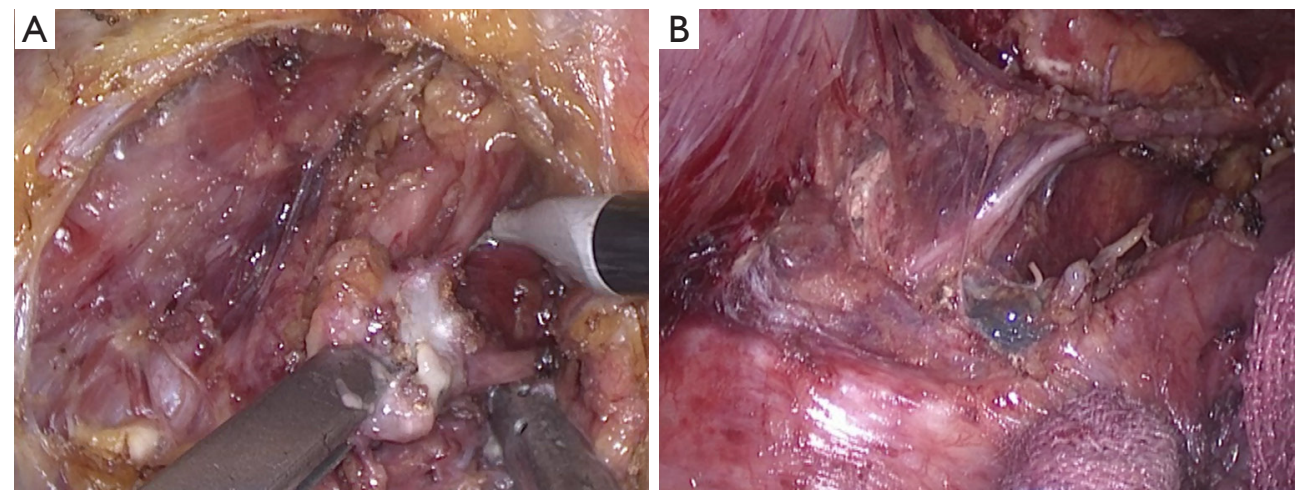

Figure 3 Intraoperative images of video-assisted thoracoscopic MIE under ergonomic port design in dissecting lymph nodes along RLN. (A) Dissecting right RLN lymph nodes; (B) dissecting left RLN lymph nodes. MIE, minimally invasive esophagectomy; RLN, recurrent laryngeal nerve.

distal to proximal just below the right main bronchus. Finally, the subcarinal lymph nodes were en bloc resected along with the esophagus after they were dissected rightly below the carina.

After the en bloc dissection of subcarinal lymph nodes, the esophagus was taped with a band for retraction of the esophagus to facilitate downward mobilization of the esophagus and dissection of lymph nodes. Finally, the thoracic part of the esophagus and surrounding lymph nodes were completely dissected.

\section{Surgical techniques in the abdomen and anastomosis}

The following procedures in the abdomen and approach for anastomosis were the same for patients in each group. Our port design for abdominal part for both groups was described in detail in our previous study (10). After freeing the stomach and completion of lymphadenectomy in the abdomen, we created a 4-cm-wide gastric tube with a linear stapling device (Johnson \& Johnson) with the stomach pulling out through an additional small upper midabdominal incision (10). Finally, we performed a layered hand-sewing esophagogastric anastomosis in the left neck for patients in each group.

\section{Statistical analysis}

Data were presented as number and (\%) for categorical data or mean \pm standard deviation for continuous variables. Student's test or Mann-Whitney U test was applied to compare continuous variables, depending on normality of distribution; while for categorical data, chi-square or Fisher's exact test was applied. All statistical analyses were 
Table 1 Comparison of baseline characteristics of patients in ergonomic group and conventional group

\begin{tabular}{|c|c|c|c|}
\hline Characteristics & Ergonomic group $(\mathrm{n}=35)$ & Conventional group $(n=35)$ & $\mathrm{P}$ \\
\hline Gender (male/female) & $25 / 10$ & $27 / 8$ & 0.584 \\
\hline Height (mean $\pm \mathrm{SD}, \mathrm{cm})$ & $163.2 \pm 6.4$ & $163.5 \pm 5.0$ & 0.934 \\
\hline Weight (mean $\pm \mathrm{SD}, \mathrm{kg}$ ) & $58.4 \pm 8.9$ & $58.1 \pm 7.8$ & 0.676 \\
\hline Hypertension & $2(5.7)$ & $4(11.4)$ & $0.393^{\#}$ \\
\hline Coronary artery disease & $3(8.6)$ & $4(11.4)$ & $0.690^{\#}$ \\
\hline Diabetes & $2(5.7)$ & $1(2.9)$ & $0.555^{\#}$ \\
\hline COPD & $5(14.3)$ & $9(25.7)$ & 0.232 \\
\hline II & $21(60.0)$ & $19(54.3)$ & \\
\hline III & $8(22.9)$ & $9(25.7)$ & \\
\hline pTNM, n (\%) & & & 0.860 \\
\hline 1 & $4(11.4)$ & $5(14.3)$ & \\
\hline II & $12(34.3)$ & $13(37.1)$ & \\
\hline III & $18(51.4)$ & $15(42.9)$ & \\
\hline IVa & $1(2.9)$ & $2(5.7)$ & \\
\hline Number of LN dissected (mean \pm SD) & $18.3 \pm 7.8$ & $17.1 \pm 7.5$ & 0.543 \\
\hline
\end{tabular}

\#, Fisher's exact test. SD, standard deviation; COPD, chronic obstructive pulmonary disease; LN, lymph node.

performed using IBM SPSS software (version 22.0; IBM Corp., Armonk, NY, USA). A two-side P value of $<0.05$ was considered statistically significant.

\section{Results}

The flow diagram of our pilot study was shown in Figure 1. Finally, a total of 70 patients were randomly assigned into two groups (ergonomic pot group and conventional port group). There were 35 patients undergoing esophagectomy under ergonomic port design and another 35 patients under conventional port design. The baseline characteristics of the two groups were presented and compared in Table 1. There was no significant difference of age $(\mathrm{P}=0.453)$, gender ( $\mathrm{P}=0.584)$, height $(\mathrm{P}=0.934)$, weight $(\mathrm{P}=0.676)$, comorbidity $(\mathrm{P}=0.314)$ as well as clinical TNM stage $(\mathrm{P}=0.889)$ between the two groups. Moreover, there was also no significant difference of pathologic TNM stage $(\mathrm{P}=0.860)$, number of total dissected lymph nodes $(\mathrm{P}=0.543)$ and positive lymph nodes $(\mathrm{P}=0.942)$ as well as total dissected mediastinal lymph nodes $(\mathrm{P}=0.280)$ between the two groups. As for perioperative outcomes, there was no significant difference of in-operating time $(\mathrm{P}=0.283)$ and blood loss $(\mathrm{P}=0.651)$ in the thoracic part between the two groups (Table 2). However, there were significantly less times of forced pause of the surgeon by fatigue during thoracic part in the ergonomic group compared to conventional group (mean time: 1.1 vs. 7.4, respectively; $\mathrm{P}<0.001)$. Moreover, the symptom score referable to the musculoskeletal system by the surgeon was significantly lower in the ergonomic group than in the conventional group (2.3 vs. 7.6; $\mathrm{P}<0.001)$. Postoperatively, there was no significant difference of 
Table 2 Comparison of short-term outcomes between ergonomic group and conventional group

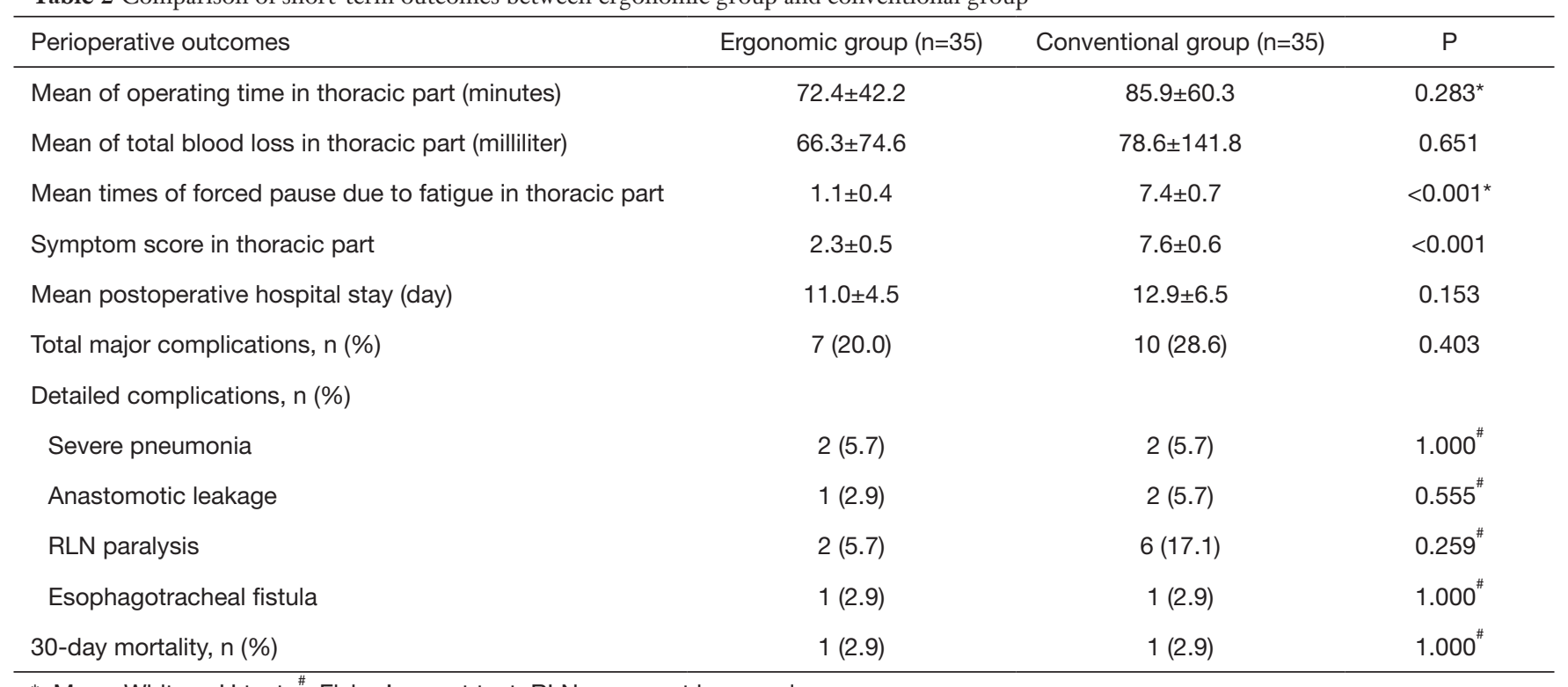

*, Mann-Whitney U test; ", Fisher's exact test. RLN, recurrent laryngeal nerve.

hospital stay $(\mathrm{P}=0.153)$, rate of complications $(\mathrm{P}=0.403)$, and 30-day mortality rate $(\mathrm{P}=1.000)$ between the two groups.

\section{Discussion}

Currently, thoracoscopic esophagectomy still imposes difficulties for thoracic surgeons due to its technical challenges and meticulous procedures $(5,7)$. Moreover, thoracoscopic esophagectomy often requires longer operation time leading to unavoidable stress for surgeons (11). The workload of surgeons undertaking thoracoscopic esophagectomy with lymphadenectomy could also cause considerable fatigue (6), and therefore, ergonomic design was badly needed to make surgeons comfortable and efficient. Currently reported procedures of thoracoscopic esophagectomy all required that the surgeons lifted their shoulders and arms and the surgeon's shoulders were kept in abduction with both arms flexed, keeping a relatively fixed gesture for a relatively long-time during operation, which could cause significant muscle fatigue and make the surgeons tired. Even though adopting the lateral-prone position was initially proved to be relatively ergonomic compared with conventional left lateral or prone position, surgeons were still required to lift their shoulders and arms $(8,12)$. In a survey of thoracic surgeons, the majority of them complained of having various degrees of discomfort mainly in the neck, shoulder and back during thoracoscopic surgery (13). Therefore, ergonomics in thoracoscopic thoracic surgery were badly needed. Hence, in this randomized comparative study, we introduced our novel ergonomic port design for thoracic part of MIE as well as our novel procedures of lymphadenectomy via tissue interactive retraction. We compared the effects of ergonomic port design with that of conventional design among a total of 70 randomly assigned patients. The baseline characteristics of the two groups were balanced and comparable. Ergonomic port design yielded at least similar degree of lymphadenectomy as well as similar postoperative complications to conventional port design. Moreover, ergonomic port design could significantly reduce shoulder fatigue compared to conventional port design and our novel procedures of lymphadenectomy via tissue interactive retraction could also facilitate the procedure of lymphadenectomy in the thoracic part. Therefore, our novel ergonomic port design and procedures of lymphadenectomy via tissue interactive retraction was proved to be feasible and could widely applied by most of the thoracic surgeons.

Compared to conventional port design which was widely applied in China, our ergonomic port design could significantly reduce the fatigue of surgeons during freeing the esophagus and lymphadenectomy in the thorax, for the fact that the main working ports were set at the third ICS and the fifth ICS around the posterior axillary line, which were close and parallel to each other, and in this case, the 
surgeon could put down his hands and did not need to lift his arms with his shoulders kept in adduction throughout the procedure avoiding muscle fatigue, which showed ergonomic benefits for the surgeon. Moreover, difficulty still existed in how to mobilize the esophagus as well as performing lymphadenectomy easily and efficiently, and therefore, improvement of those procedures still needed further research (14). Our procedures of mobilization of the esophagus and lymphadenectomy by utilizing the tissue interactive retraction could make thoracoscopic esophagectomy easier and more efficient. For example, at the very beginning, the arch of azygos vein was ligated at the conjugation of azygos vein and superior intercostal vein and the azygos vein and right superior intercostal vein as well as the arch of azygos vein were clipped respectively without any risk of bleeding, which provided excellent surgical field and grasping point for mobilization of the esophagus. For the dissection of left mediastinal lymph nodes, the esophagus and trachea were pulled laterally and anteriorly via the interactive retraction force between the esophagus and trachea to provide excellent surgical field for easy dissection avoiding injury to the left recurrent nerve. The subcarinal lymph nodes were en bloc resected along with the esophagus from left to right via the interactive retraction of the esophagus and the lymph nodes when pulling the esophagus laterally and then posteriorly, which eased the procedure significantly. In conclusion, our initial experience confirmed those advantages of the ergonomic thoracoscopic esophagectomy with lymphadenectomy in the thoracic phrase.

However, our study still had several limitations. First, this study suffered from the limitation of relatively small sample size, which may limit our analytical power. Second, application of subjective parameter in the ergonomics evaluation could also limit the validity of our results. Moreover, we only presented our initial preliminary results from the pilot study in one surgical team, which may limit the generalization of our conclusions. Therefore, further well-conducted multicenter randomized-controlled trials are needed to confirm and update our conclusions.

\section{Conclusions}

Our novel ergonomic port design for thoracoscopic esophagectomy as well as lymphadenectomy via tissue interactive retraction was proved to be safe and viable for ergonomically freeing the esophagus and completing lymphadenectomy in the thorax, which could be mastered easily by most of the thoracic surgeons. Further studies are needed to confirm and update our conclusions.

\section{Acknowledgments}

Funding: This work was supported by National Natural Science Foundation of China (to Yi-Dan Lin; No.81672291, No.31071210).

\section{Footnote}

Conflicts of Interest: The authors have no conflicts of interest to declare.

Ethical Statement: The authors are accountable for all aspects of the work in ensuring that questions related to the accuracy or integrity of any part of the work are appropriately investigated and resolved. Our work was approved by institutional review board of West China Hospital, Sichuan University (No. 20170730) and informed consent was obtained from each patient participating in the study.

\section{References}

1. Pennathur A, Gibson MK, Jobe BA, et al. Oesophageal carcinoma. Lancet 2013;381:400-12.

2. Biere SS, van Berge Henegouwen MI, Maas KW, et al. Minimally invasive versus open oesophagectomy for patients with oesophageal cancer: a multicentre, open-label, randomised controlled trial. Lancet 2012;379:1887-92.

3. Yerokun BA, Sun Z, Jeffrey Yang CF, et al. Minimally Invasive Versus Open Esophagectomy for Esophageal Cancer: A Population-Based Analysis. Ann Thorac Surg 2016;102:416-23

4. Rodham P, Batty JA, McElnay PJ, et al. Does minimally invasive oesophagectomy provide a benefit in hospital length of stay when compared with open oesophagectomy? Interact Cardiovasc Thorac Surg 2016;22:360-7.

5. Okamura A, Watanabe M, Mine S, et al. Factors influencing difficulty of the thoracic procedure in minimally invasive esophagectomy. Surg Endosc 2016;30:4279-85

6. Shen Y, Feng M, Tan L, et al. Thoracoscopic esophagectomy in prone versus decubitus position: ergonomic evaluation from a randomized and controlled study. Ann Thorac Surg 2014;98:1072-8.

7. Ozawa S, Ito E, Kazuno A, et al. Thoracoscopic 
esophagectomy while in a prone position for esophageal cancer: a preceding anterior approach method. Surg Endosc 2013;27:40-7.

8. Li X, Lai FC, Qiu ML, et al. Minimally Invasive Esophagectomy in the Lateral-prone Position: Experience of 226 Cases. Surg Laparosc Endosc Percutan Tech 2016;26:60-5.

9. Zhang H, Chen L, Geng Y, et al. Modified anastomotic technique for thoracolaparoscopic Ivor Lewis esophagectomy: early outcomes and technical details. Dis Esophagus 2017;30:1-5.

10. Deng HY, Huang WX, Li G, et al. Comparison of shortterm outcomes between robot-assisted minimally invasive esophagectomy and video-assisted minimally invasive esophagectomy in treating middle thoracic esophageal cancer. Dis Esophagus 2018. doi: 10.1093/dote/doy012.

Cite this article as: Deng HY, Zheng X, Alai G, Zhuo ZG, Li G, Luo J, Lin YD. Ergonomic thoracic port design for videoassisted thoracoscopic minimally invasive esophagectomy and lymphadenectomy: a preliminary pilot study. Ann Transl Med 2019;7(22):679. doi: 10.21037/atm.2019.08.19
11. Yoshida N, Baba Y, Shigaki H, et al. Effect of Esophagus Position on Surgical Difficulty and Postoperative Morbidities After Thoracoscopic Esophagectomy. Semin Thorac Cardiovasc Surg 2016;28:172-9.

12. Ma Z, Niu H, Gong T. Thoracoscopic and laparoscopic radical esophagectomy with lateral-prone position. J Thorac Dis 2014;6:156-60.

13. Welcker K, Kesieme EB, Internullo E, et al. Ergonomics in thoracoscopic surgery: results of a survey among thoracic surgeons. Interact Cardiovasc Thorac Surg 2012;15:197-200.

14. Xi Y, Ma Z, Shen Y, et al. A novel method for lymphadenectomy along the left laryngeal recurrent nerve during thoracoscopic esophagectomy for esophageal carcinoma. J Thorac Dis 2016;8:24-30. 